\title{
TIME-DEPENDENT SIMULATION OF OBLIQUE MHD COSMIC-RAY SHOCKS USING THE TWO-FLUID MODEL
}

\author{
ADAM Frank AND T. W. JoNES \\ Department of Astronomy, University of Minnesota, Minneapolis, MN 55455 \\ AND \\ DONGSU RYU \\ Department of Astronomy and Space Science, Chungnam National University, Daejeon 305-764, Korea \\ Received 1993 October 18; accepted 1994 September 22
}

\begin{abstract}
Using a new, second-order accurate numerical method we present dynamical simulations of oblique MHD cosmic-ray (CR)-modified plane shock evolution. Most of the calculations are done with a two-fluid model for diffusive shock acceleration, but we provide also comparisons between a typical shock computed that way against calculations carried out using the more complete, momentum-dependent, diffusion-advection equation. We also illustrate a test showing that these simulations evolve to dynamical equilibra consistent with previously published steady state analytic calculations for such shocks.

In order to improve understanding of the dynamical role of magnetic fields in shocks modified by CR pressure we have explored for time asymptotic states the parameter space of upstream fast mode Mach number, $M_{f}$, and plasma $\beta$. We compile the results into maps of dynamical steady state CR acceleration efficiency, $\epsilon_{c}$. Since the models are simplifications, such maps should not be used to predict quantitatively $\epsilon_{c}$ in real shocks; however, they are internally consistent, so that they can enable us to compare various competing dynamical effects. The maps, along with additional numerical experiments, show that $\epsilon_{c}$ is reduced through the action of compressive work on tangential magnetic fields in CR-MHD shocks. Thus $\epsilon_{c}$ in low $\beta$, moderate $M_{f}$ shocks tends to be smaller in quasi-perpendicular shocks than it would be for high $\beta$ shocks of the same $M_{f}$. This result supports earlier conclusions that strong, oblique magnetic fields inhibit diffusive shock acceleration. For quasi-parallel shocks with $\beta<1$, on the other hand, $\epsilon_{c}$ seems to be increased at a given $M_{f}$ when compared to high $\beta$ shocks. The apparent contradiction to the first conclusion results, however, from the fact that for small $\beta$ quasi-parallel shocks, the fast mode Mach number is not a good measure of compression through the shock. That is better reflected in the sonic Mach number, which is greater in these instances. Acceleration efficiencies for high and low $\beta$ having comparable sonic Mach numbers are more similar.

Time evolution of CR-MHD shocks is qualitatively similar to CR-gasdynamical shocks. However, several potentially interesting differences are apparent. We have run simulations using constant, and nonisotropic, obliquity (and hence spatially) dependent forms of the diffusion coefficient $\kappa$. Comparison of the results shows that while the final steady states achieved are the same in each case, the history of CR-MHD shocks can be strongly modified by variations in $\kappa$ and, therefore, in the acceleration timescale. Also, the coupling of CR and MHD in low $\beta$, oblique shocks substantially influences the transient density spike that forms in strongly CRmodified shocks. We find that inside the density spike a MHD slow mode wave can be generated that eventually steepens into a shock. A strong shear layer develops within the density spike, driven by MHD stresses. We conjecture that currents in the shear layer could, in nonplanar flows, result in enhanced particle accretion through drift acceleration.
\end{abstract}

Subject headings: acceleration of particles - cosmic rays - MHD - shock waves

\section{INTRODUCTION}

In the past several years nonlinear theories of diffusive shock acceleration have demonstrated the potential importance of cosmic-ray (CR) dynamical feedback on the evolution of shock structures (see reviews by Blandford \& Eichler 1987; Berezhko \& Krymskii 1988; Jones \& Ellison 1991). A range of methods has been successfully applied to show that CR pressures can become sufficient to modify shock structures substantially (e.g., Drury \& Völk 1981; Achterberg, Blandford, \& Periwal 1984; Ellison \& Eichler 1984; Webb, Drury, \& Völk 1986; Falle \& Giddings 1987; Kang \& Jones 1990, 1991). In some cases the modifications can even produce smooth CR-dominated shocks with no entropy-generating gas subshock. Numerical simulations using a variety of techniques have also demonstrated the importance of time-dependent effects in the determination of CR-modified shock properties (e.g., Drury \& Falle 1987; Falle \& Giddings 1989; Kang \& Jones 1991; Duffy 1992; Kang, Jones, \& Ryu 1992, Ryu, Kang, \& Jones 1993). This point refers first to the fact that, for many reasons, CR shock transitions may not be stationary structures. It also has been noted that in evolving toward an apparent equilibrium structure, shocks can produce nonequilibrium structures that persist downstream of the shock even after the shock itself has stopped evolving significantly.

Through all of this the presence of a large-scale magnetic field was generally understood to be an essential physical ingredient in CR shocks, since magnetic field fluctuations (small-scale Alfvén waves, or "Alfvénic turbulence" to avoid 
confusion with large-scale waves) mediate the $\mathrm{CR}$ propagation. But the magnetic fields are rather difficult to treat explicitly and fully, especially when those fields play a direct dynamical role. So, most studies of CR-modified shocks have focused on pure gasdynamical models of the flows. That may be adequate if the fields are sufficiently weak, and/or the large-scale field is aligned parallel to the shock normal, so that sonic-mode shock dynamics applies. Except for modeling the spatial dependence of the CR diffusion coefficient, explicit treatment of the magnetic field may not always be generally necessary when the shock speed in the preshock (upstream) fluid, $u_{s}$, and the preshock sound speed, $a$ are large compared to the preshock Alfvén speed, $b$. The second of these conditions is commonly expressed as $\beta=a^{2} / b^{2} \gg 1$. But, this condition is likely to be violated, perhaps strongly, in many environments where particle acceleration occurs (e.g., Slavin \& Cox 1992). Even if the magnetic field and shock normal are parallel, treatment of $C R$ shocks in flows with $\beta \lesssim 1$ may depend in significant ways on $\beta$, especially if the Alfvénic Mach number is not very large. One must be concerned, for example, with the finite momentum and energy carried by the CR-mediating Alfvénic turbulence (e.g., Völk, Drury, \& McKenzie 1984; Jones 1993).

Postponing for now discussion of the more complicated topics in general MHD flows, our purpose in the present paper is to examine some straightforward dynamical influences of oblique magnetic fields on plane CR shocks. There are several obvious ways in which oblique fields may be important. If the pressure in the tangential field is comparable to the gas pressure of the incoming flow, that changes the jump conditions to be satisfied through the shock and must, therefore, influence the transfer of energy and momentum of CRs within the shock (e.g., Webb 1983). The anisotropic nature of MHD stresses in oblique shocks leads to the existence of fast, intermediate, and slow wave mode families, each with distinct behaviors. That can complicate the shock properties and lead to the generation of distinctly MHD time-dependent structures in a shock evolving in response to build up of CR pressure. This increase in the degrees of freedom at the shock also augments the modes through which the shock structure may become dynamically unstable (e.g., Chalov 1988; Zank, Axford, \& McKenzie 1990). The CR diffusion coefficient may be anisotropic relative to the field, affecting the rates at which CRs are accelerated. If the diffusion is described by standard kinetic theory models, the acceleration rate at oblique shocks could be much faster than at otherwise comparable parallel shocks (Jokipii 1987; Ostrowski 1988); however, the resulting time-asymptotic testparticle form for the particle spectrum is apparently unchanged (Bell 1978), at least for nonrelativistic shocks (Kirk \& Heavens 1989). This change in the acceleration rate should influence the dynamical properties of the shock at intermediate times, before a dynamical equilibrium is achieved, even if the field is weak and not directly involved dynamically. On the other hand, Baring, Ellison, \& Jones (1993) have shown that the efficiency of thermal particle injection into the CR population can be decreased by perpendicular magnetic field components. It is clearly important to begin the process of understanding better the roles that these various processes might play in astrophysical settings. The most broadly applicable way to include magnetic fields explicitly in CR shock studies is through fully time-dependent MHD simulations. The purpose of the present paper is to take a first step in that direction. We will outline our method of doing that in the following section.

\section{METHODS}

\subsection{Diffusive Cosmic-Ray Transport}

The standard starting point for treating the transport of CR around shocks is the "diffusion-advection" equation, (e.g., Parker 1965; Skilling 1975). For plane symmetric flows with the plane normal in the $x$-direction in which second-order, momentum diffusion, and Alfvénic turbulence drift can be neglected, this can be written

$$
\frac{\partial f}{\partial t}+\frac{\partial}{\partial x}\left(u_{x} f-\kappa \frac{\partial f}{\partial x}\right)=\frac{1}{3 p^{2}}\left(\frac{\partial p^{3} f}{\partial p}\right) \frac{\partial u_{x}}{\partial x}+Q
$$

where $f(x, p, t)$ is the isotropic part of the momentum distribution, $u_{x}$ is the fluid velocity in the $x$-direction, $p$ is the particle momentum, and $\kappa$, usually called the diffusion coefficient, is the spatial CR diffusion tensor projected along the $x$-direction. $Q$ is a source term that can be used to allow injection of particles from the thermal plasma into the (more energetic) CR particle population or escape at high energies, for example. Although equation (2.1) was originally derived for " quasi-parallel " flows, in which the magnetic field lies in the $x$-direction, it has been demonstrated to be valid for oblique field geometries as well, provided, there is diffusion along the shock normal and flows are truly plane-symmetric (Jokipii 1982; Webb, Axford, \& Terasawa 1983). Complete specification of the problem using equation (2.1) requires, in addition, equations defining the dynamics of the base fluid, such as we will prescribe in the following subsection. If the CR pressure, $P_{c}$, is dynamically important it must be included in those equations, of course (e.g., Achterberg 1982). In general, one assumes in treatments of this kind that the inertia of the CR particles is insignificant, so that only the momentum and energy exchanges between thermal plasma and CR matter dynamically.

Because equation (2.1) must be solved on a momentum grid, $p$, as well as a spatial grid, it is computationally a rather expensive tool to be used for evolving MHD CR shocks long enough to achieve something close to an equilibrium structure. Our present primary intent is to provide a first, broad exploration of the dynamic evolution of MHD-CR flows, as a follow-up and confirmation of some previous steady state, analytical calculations. Use of equation (2.1) would be prohibitively expensive for that task. Fortunately, it is possible to address these dynamical questions more economically. Thus, for most of our calculations we will employ a simpler approach. The dynamical content of the diffusion-advection equation is contained in its energy moment; namely the CR energy conservation equation (e.g., Drury \& Völk 1981; Achterberg 1982; Drury 1983; Kang \& Jones 1990),

$$
\frac{\partial E_{c}}{\partial t}+\frac{\partial}{\partial x}\left(u_{x} E_{c}-\langle\kappa\rangle \frac{\partial E_{c}}{\partial x}\right)=-P_{c} \frac{\partial u_{x}}{\partial x}+S_{e}
$$

In equation (2.2) $E_{c}$ is the energy density in CR particles. The source term, $S_{e}$, is a closure parameter related to $Q$. Suitable definitions of $P_{c}$ and $E_{c}$ can be found, for example, in Kang \& Jones (1990). The energy-weighted, mean diffusion coefficient, $\langle\kappa\rangle$, is defined as

$$
\langle\kappa\rangle=\frac{\int_{p_{0}}^{p_{1}} \kappa \partial f / \partial x\left(\sqrt{p^{2}+1}-1\right) p^{2} d p}{\int_{p_{0}}^{p_{1}} \partial f / \partial x\left(\sqrt{p^{2}+1}-1\right) p^{2} d p},
$$

where $p$ is expressed in units of $m c$. The third closure parameter needed for equation (2.2) is the adiabatic index, $\gamma_{c}=1+P_{c} / E_{c}$, for the CR "fluid." It falls in the range $4 / 3 \leq \gamma_{c} \leq 5 / 3$, depend- 
ing on the portion of $\mathrm{CR}$ particles that are ultrarelativistic or nonrelativistic. Although applications of equation (2.2) commonly assume both $\langle\kappa\rangle$ and $\gamma_{c}$ to be constants, that is certainly not necessary.

Since equation (2.2) represents the CR through bulk properties, its combination with the ordinary fluid equations to represent the "thermal" plasma is usually called the two-fluid model for CR transport. With complete specification of the three closure parameters $\left(S_{e},\langle\kappa\rangle\right.$, and $\left.\gamma_{c}\right)$, it must give exactly the same solutions as treatments based on the full diffusionadvection equation, (2.1). These three parameters are clearly functions of the solution, however, so ordinarily two-fluid solutions must be considered approximate relative to the more complete diffusion-advection solutions. Because of this there has been some criticism of the model's use (e.g., Achterberg et al. 1984; Jones \& Ellison 1991). On the other hand, a number of studies have shown rather good qualitative and even semiquantitative agreement between diffusion-advection calculations and two-fluid calculations involving quasi-parallel flows and simple models for the behavior of the closure parameters (e.g., Falle \& Giddings 1987; Kang \& Jones 1991, 1995; Kang et al. 1992; Duffy 1992; Duffy et al. 1993, 1994). We will revisit this issue for the present context and provide a comparison of calculations done both ways in $\S 3.1$.

Webb (1983) first applied two-fluid methods to equilibrium, steady state CR-MHD modified shock structures for the case where the upstream gas is cold (its pressure can be ignored) and the magnetic field is oblique. Webb's steady analytic calculations demonstrated that, as for the $\mathrm{CR}$ modified gasdynamical flows, both subshock-containing and smoothed, CR-dominated solutions to the CR-MHD shock equations were possible. However, his solutions suggested that shock CR acceleration was less effective when the upstream tangential component of the field was strong. Subsequently Kennel, Edmiston, \& Hada (1985) and Webb et al. 1986 (hereafter WDV) calculated steady state two-fluid CR-MHD modified shock structures with finite upstream gas pressures. The WDV analysis, which included $\beta=5$ and $\beta=1$ cases and a range of fast mode Mach numbers, $M_{f}$, lead those authors to conclude that the efficiency of CR acceleration was suppressed when the obliquity of the upstream magnetic field was high. They also found that the reduction in CR acceleration efficiency was most pronounced in the lower $\beta(\beta=1)$ flows of low, fast-mode Mach number $\left(M_{f}<6\right)$. For high $\beta(\beta=5)$ flows the CR acceleration efficiency was only weakly affected by changes in the upstream magnetic field angle regardless of the fast-mode Mach number.

The time-dependent work reported in this paper is intended as a first extension of the previous analytical work. For the reasons cited earlier we will postpone extensive use of the full diffusion-advection equation, (2.1), but will present primarily the results of two-fluid computations. In an earlier work (Frank, Jones, \& Ryu 1993) we confirmed Webb's cold gas solutions, presented preliminary results from more general CR-MHD flows, and determined the convergence properties of our numerical method. In the present paper we explore the time evolution of finite gas pressure $\left(P_{g} \neq 0\right)$ models of CR-MHD modified oblique shocks and their relation to the WDV steady state calculations. We will also explore in more detail questions raised in WDV concerning the role of both the obliquity of the field and the tangential field strength directly in determining the $\mathrm{CR}$ acceleration efficiency. More general timedependent effects unique to CR-MHD modified shocks will also be examined along with their potential importance for $\mathrm{CR}$ studies. As in WDV, we will concentrate on those flows with high enough Alfvénic Mach number that switch-on and switch-off shocks do not occur. In the regime where those special shocks form, CR scattering centers (the Alfvénic turbulence) move at speeds comparable to the gas, requiring that the time-dependent wave field dynamics be included in the calculations. For the present we restrict ourselves to flows where the scattering centers can be considered moving with the fluid. Jun, Clarke, \& Norman (1994) have also recently simulated CR-MHD shocks using two-fluid methods. Their results are complementary to ours, since they restricted their analysis to the special case of perpendicular (magnetosonic) shocks. Consequently, they could not explore a number of questions address here.

The remainder of the paper is organized as follows. Section 2 introduces the fundamental equations governing CR modified MHD flows and discusses their application to our methods. An appendix at the end discusses these numerical methods in more detail. Section 3 briefly addressed the behavior of twofluid methods for this study through some tests against solutions given in WDV and direct comparison with our own diffusion-advection simulations. Section 4 explores issues concerning the dependence of CR shock acceleration on upstream magnetic field properties. In $\S 5$ we examine the general timedependent features of CR-MHD modified shocks as seen in our simulations. We provide in $\S 6$ a discussion of these results in light of previous studies and their potential for application in higher dimensional flows. Our conclusions are given in $\$ 7$.

\subsection{Numerical Methods}

In addition to equation (2.1) or (2.2) we solve the equations of nonrelativistic ideal MHD for one-dimensional flow in Cartesian coordinates (e.g., Jeffrey 1986). As with gasdynamical models the conservation equations are modified to include momentum and energy source terms from CR feedback (e.g., Drury \& Völk 1981; Jones \& Kang 1990). The MHD equations are written in conservative, vector form as

$$
\frac{\partial \boldsymbol{q}}{\partial t}+\frac{\partial \boldsymbol{F}}{\partial x}=\boldsymbol{S}
$$

with

$$
q=\left(\begin{array}{l}
\rho \\
\rho u_{x} \\
\rho u_{y} \\
\rho u_{z} \\
B_{y} \\
B_{z} \\
E
\end{array}\right),
$$

and

$$
F=\left(\begin{array}{c}
\rho u_{x} \\
\rho u_{x}^{2}+P^{*}-B_{x}^{2} \\
\rho u_{x} u_{y}-B_{x} B_{y} \\
\rho u_{x} u_{z}-B_{x} B_{z} \\
B_{y} u_{x}-B_{x} u_{y} \\
B_{z} u_{x}-B_{x} u_{z} \\
\left(E+P^{*}\right) u_{x}-B_{x}\left(B_{x} u_{x}+B_{y} u_{y}+B_{z} u_{z}\right)
\end{array}\right)
$$


while the CR source term vector is

$$
\boldsymbol{S}=-\left(\begin{array}{c}
0 \\
\partial P_{c} / \partial x \\
0 \\
0 \\
0 \\
0 \\
u_{x} \partial P_{c} / \partial x+S_{e}
\end{array}\right)
$$

The CR pressure, $P_{c}$, along with $S_{e}$ are derived from either equation (2.1) or (2.2). In these relations

$$
\begin{gathered}
P^{*}=P_{g}+\frac{1}{2}\left(B_{x}^{2}+B_{y}^{2}+B_{z}^{2}\right)=P_{g}+\frac{1}{2} B^{2} \\
E=\frac{1}{2} \rho\left(u_{x}^{2}+u_{y}^{2}+u_{z}^{2}\right)+\frac{1}{\left(\gamma_{g}-1\right)} P+\frac{1}{2}\left(B_{x}^{2}+B_{y}^{2}+B_{z}^{2}\right) .
\end{gathered}
$$

The magnetic field components are expressed in rationalized units

$$
B \rightarrow \frac{B}{\sqrt{4 \pi}},
$$

so that the Alfven speed, $b=B /(\rho)^{1 / 2}$. The following definitions hold: $\rho$ is the mass density; $u_{x}, B_{x}$, and $u_{y}, u_{z}, B_{y}, B_{z}$ are the components of velocity and magnetic field parallel and perpendicular to shock front; $\boldsymbol{P}_{\boldsymbol{g}}$ and $\gamma_{g}$ are the gas pressure and adiabatic index.

In the interest of simplicity we will not consider here flows with rotations of the shock-normal/magnetic-field plane between the initial upstream and downstream states, although the numerical code is quite capable of handling such features. Thus, without further loss of generality we can place the magnetic field in the $X-Z$ plane, $B=\left(B_{x}, 0, B_{z}\right)=B(\cos \theta, 0$, sin $\theta)$. We will also select a reference frame so that there is no upstream tangential velocity; $u_{y}=u_{z}=0$. All of the simulations discussed here are piston-driven shock tubes. We establish the flows by projecting magnetized fluid with embedded $\mathrm{CR}$ in from the right boundary, using continuous boundary conditions and reflecting it off a wall (piston) at the left boundary. The tangential magnetic field at the left boundary is "mirrored" in a manner analogous to the gas density. Thus there is no current sheet on the piston surface. In other words, the simulations can be considered and those of two identical, colliding clouds with magnetic field "continuous" across them. The CR pressure is given continuous boundary conditions on both ends of the grid.

In general, spatial CR diffusion will differ in directions parallel and perpendicular to the magnetic field. Diffusive acceleration depends on the diffusion along the shock normal, since that determines the rate at which particles recross the shock. Thus, the appropriate diffusion coefficient in equations (2.1)(2.2) will take the form (e.g., Drury 1983; Webb 1983)

$$
\kappa=\kappa_{\|} \cos ^{2} \theta+\kappa_{\perp} \sin ^{2} \theta
$$

where the directions $\|$ and $\perp$ refer to the magnetic field direction. Note, of course, that $\theta$ is generally a changing function of space and time. In standard kinetic theory scattering models of particle diffusion the ratio $\kappa_{\|} / \kappa_{\perp} \sim 1+(\omega \tau)^{2}$, where $\omega$ and $\tau$ are the particle gyro frequency and collision time, respectively (e.g., Jokipii 1987; Zank et al. 1990). We will adopt the form for $\kappa$ in equation (2.11) in our simulations.

Previous studies of CR-modified shocks have shown that the time required to evolve toward dynamical equilibrium scales with the so-called diffusion time, $t_{d}$ (e.g., Drury \& Falle 1986; Jones \& Kang 1990), which in the present case is conveniently expressed as

$$
t_{d}=\frac{\langle\kappa\rangle}{u_{s}^{2}}=\frac{x_{d}}{u_{s}},
$$

where $u_{s}$ is the shock speed in the upstream fluid (see eq. [3.6]) and $x_{d}$ is known as the diffusion length. The quantity $x_{d}$ is a measure of the breadth of the CR precursor to the shock. It is important that computational resolution be fine enough to resolve the flow features on this scale. For our discussion we define, therefore, the resolution ratio of each simulation to be

$$
n_{r}=\frac{x_{d}}{\Delta x}
$$

where $\Delta x$ is the size of a computational zone. Frank et al. (1993) found that provided $n_{r}>10-20$, our code produced accurate and converged solutions. When $\left\langle\kappa_{\|}\right\rangle \neq\left\langle\kappa_{\perp}\right\rangle, t_{d}$ is not well defined, since $\langle\kappa\rangle$ changes through a shock. To be specific, however, we will adopt the convention of using the value of $\langle\kappa\rangle$ from equation (2.11) upstream of the shock to define $t_{d}$.

Our numerical method solves equation (2.4) through the second-order accurate, Eulerian finite difference, Total Variation Diminishing (TVD) scheme. The TVD scheme was originally designed for gasdynamics by Harten (1983) and extended to MHD by Brio \& Wu (1988). In this paper, we use the version of the MHD-TVD code which was subsequently improved and fully tested by Ryu \& Jones (1995). The pure $\operatorname{MHD}(S=0)$ form of the equation is solved with the aid of an approximate MHD Riemann solver used to estimate the flux, $\boldsymbol{F}$. CR source corrections, $\boldsymbol{S}$, are added separately in a manner preserving second-order accuracy. Shocks and other discontinuities are generally sharply resolved within a few zones. The CR diffusion-advection or energy equation is solved separately using a second-order combined Lagrangian Crank-Nicholson and monotone remap scheme. The mixed scheme is efficient and provided us much better stability and accuracy than a straight Eulerian Crank-Nicholson approach. In the Appendix we present the numerical method in more detail. Gasdynamical versions of the code were tested extensively against both analytical steady state solutions and numerical timedependent models calculated with our well-tested PPM twofluid and diffusion-advection codes (Jones \& Kang 1990; Kang \& Jones 1991), all with excellent agreement. The pure MHD code was tested using a nonlinear MHD Riemann solver against a variety of planar shock tube problems involving all three wave mode families (for details, see Ryu \& Jones 1994).

\section{COSMIC-RAY-MAGNETOHYDRODYNAMIC TESTS}

We also carried out a number of tests of our CR-MHD methods. Frank et al. (1994) presented tests of the two-fluid code against the steady cold plasma calculations of Webb (1983). To further validate our procedures we now illustrate two kinds of additional tests. First, we offer a comparison with the more general analytical steady state calculations of twofluid oblique CR-MHD shocks from WDV. Second, we present a comparison of the evolution of oblique CR-MHD shocks as computed from two-fluid and momentumdependent, diffusion-advection methods.

To carry out these tests we have computed the evolution of shocks responding to diffusive particle acceleration until they 
reach an approximate dynamical equilibrium. Those are the conditions we can compare to previous analytical shock properties, of course. This also serves as a confirmation that the dynamical steady state conditions can, in fact, be reached in a finite time from suitable initial conditions In addition, it gives some indications of how sensitive or insensitive these steady state flows are to the methods used to compute them.

Again, all the simulations involve flows reflected off a piston at the left boundary. To describe the flows there are four parameters needed in addition to the initial properties of the CR distribution, $f(p)$, or, in two-fluid simulations, the closure parameters $\gamma_{c}$ and $\kappa$. We can parameterize our upstream state by the following dimensionless quantities:

$$
\begin{aligned}
& \beta=\frac{a^{2}}{b^{2}}=\frac{\gamma_{g} P_{g}}{B^{2}}, \quad N=\frac{P_{c}}{P_{g}+P_{c}}, \\
& M_{f}=\frac{\left|u_{x}\right|}{c_{f}}, \quad \theta_{0}=\tan ^{-1}\left(\frac{B_{z}}{B_{x}}\right) .
\end{aligned}
$$

In equation (3.1) all quantities refer to the upstream (incoming flow) state and $u_{x}$ is measured in the computed (piston) frame. In the following discussion wherever it is necessary to distinguish upstream and downstream conditions they will receive subscripts ${ }_{1}$ and ${ }_{2}$, respectively. Of the remaining variables in equation (3.1) $a$ is the gas sound (acoustic) speed, $b$ is the Alfvén speed, $c_{f}$ is the fast-mode wave speed; namely,

$$
\begin{gathered}
a=\sqrt{\frac{\gamma_{g} P_{g}}{\rho}}, \\
c_{f}^{2}=\frac{1}{2}\left\{b^{2}+a^{2}+\left[\left(b^{2}+a^{2}\right)^{2}-4 b^{2} a^{2} \cos ^{2} \theta_{0}\right]^{1 / 2}\right\},
\end{gathered}
$$

and $\theta_{0}$ is termed the "obliquity" of the shock. Note that the fast mode Mach number, $M_{f}$, in our simulations refers to the piston rather than the shock. The equivalent shock Mach number is

$$
M_{f s}=\frac{\left|u_{s}\right|}{\left|u_{x}\right|} M_{f} .
$$

The relationship between $M_{f}$ and $M_{f s}$ depends on the compression through the shock, which is generally a function of the solution itself and a function of time as $P_{c 2}$ changes. For most of the shocks described below, $M_{f}<M_{f s} \lesssim 4 / 3 M_{f}$. Our definition of $\beta$ differs from that used frequently in the plasma physics literature and by WDV by a factor $\gamma_{g} / 2=5 / 6$. For all the simulations described, the upstream flow is characterized by $\rho=1, u_{x}=-1$, and $N=\frac{1}{2}$. For the results presented we set the source term $S_{e}=0$. We note in passing, however, that in the course of this investigation we carried out analogous calculations with $S_{e} \neq 0$ to model the injection of low-energy CRs. Behaviors of these shocks were not qualitatively different from those we will describe (cf. Kang \& Jones 1990, 1991).

\subsection{Comparison with Previous Steady State MHD Calculations}

We start with a comparison between properties of a steady state two-fluid model computed by WDV and the approximately equivalent time asymptotic flow found by us. Figure 1 illustrates the evolution to an apparent dynamical steady postshock state of a simulated two-fluid flow with upstream parameters $\beta=1, \gamma_{c}=4 / 3,\langle\kappa\rangle=0.2$, and $\theta_{0}=60^{\circ}$. The resolution ratio was $n_{r}=20$, so that the solution is well converged. The simulation was designed to match as closely as possible a solution presented by WDV in which $M_{f s}=6.1$.
(Note that their definition of $c_{f}$ included $P_{c}$ as a contributor to the sound speed. We have corrected for that in establishing an appropriate comparison.) The downstream partial pressures, $P_{c 2}, P_{g 2}$ and $P_{B 2}$, from WDV are indicated by dashed lines. The final $u_{s}$ (and thus $M_{f s}$ ) is hard to predict exactly, because it depends on $P_{c 2}, P_{g 2}$ and $P_{B 2}$ in nonlinear ways. In addition, the WDV solution was read from a published figure (Fig. 12 in DWV), so we cannot match the two problems exactly. Nonetheless, we find that all the partial pressures agree to within about $2 \%$ when normalized by the total momentum flux incident on the shock, $\bar{P}$, defined as

$$
\bar{P}=\rho_{1} u_{s}^{2}+P_{B 1}+P_{g 1}+P_{c 1},
$$

where, to estimate $u_{s}$ in the final steady shock, we assume the mass flux jump conditions are exactly satisfied across the shock. Then the required $u_{s}$ in the frame of the upstream fluid can be written in terms of jump conditions across the shock in the computed frame as

$$
u_{s}=\frac{\left[\rho u_{x}\right]}{[\rho]}-u_{x 1}=\frac{r}{r-1}\left|u_{x 1}\right|,
$$

where

$$
r=\frac{\rho_{2}}{\rho_{1}}
$$

is the compression ratio through the full shock structure.

Figure 1 shows the flow at times $t / t_{d}=200,500$, and 900 . The shock transition has approached a steady state structure by the last time shown. The rather large number of diffusion times needed for this shock to come into a dynamic equilibrium is consistent with previous gasdynamical CR shock calculations (e.g., Jones \& Kang 1990; Kang \& Jones 1991). Jones \& Kang (1990) pointed out that the rate for CR pressure to increase in response to diffusive acceleration depends not only on $t_{d}$, but also on the CR adiabatic index. Physically that derives from the fact that pressure generated by nonrelativistic particles scales as the particle momentum squared, while it increases only linearly with momentum for relativistic particles. Thus the pressure builds more slowly for relativistic CR for equal rates of momentum gain. Since $\gamma_{c}=4 / 3$ in this model, the pressure is assumed to come entirely from relativistic particles. The time to reach equilibrium also depends on the total change in $P_{c}$, of course, and so on $N$ and $M_{f s}$.

As $P_{c}$ builds within the shock precursor, a postshock density spike forms and then is left behind after the shock approaches equilibrium. The origin of this feature in strongly modified gasdynamic CR shocks was explained by Drury (1987) and independently by Jones \& Kang (1990). Briefly, it reflects an adiabatic compression in the precursor at times before an initially strong subshock weakens in response to substantial adiabatic heating in the precursor. The tangential magnetic field participates in the development of that feature in oblique MHD shocks, as can be seen in Figure 1. These features are examples of time-dependent effects that occur in the evolution of CR-modified MHD shock structures and of which the steady analytical models can give no hint. We will discuss these time-dependent features in more detail in $\S 4.5$.

\subsection{Comparison of Diffusion-Advection and Two-Fluid Solutions}

Our survey below is carried out using the two-fluid model, because it is currently too expensive to conduct such a study 

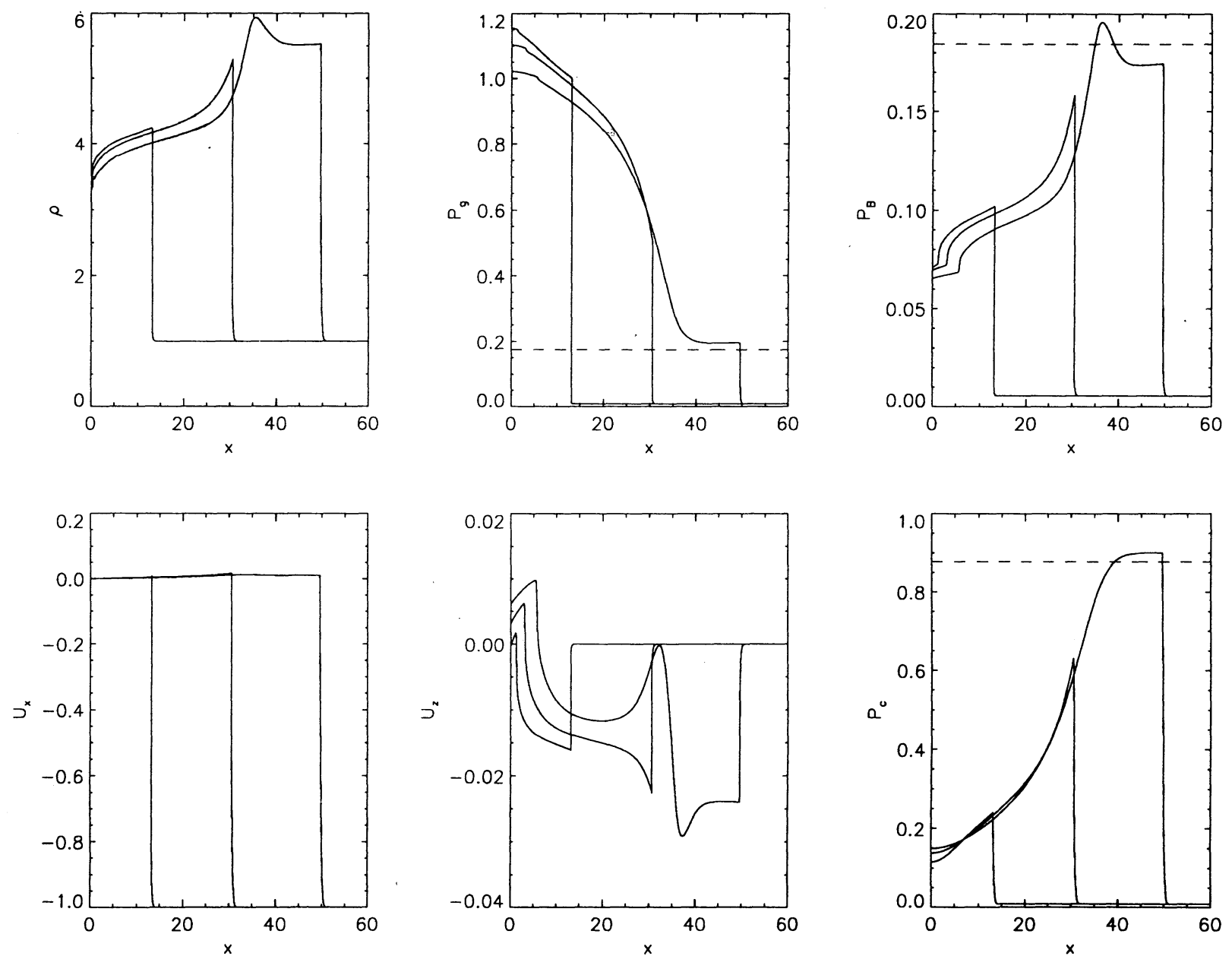

FIG. 1.-Comparison for properties of an oblique CR-MHD shock found by analytical means (postshock state shown by horizontal lines) and the results of a time-dependent two-fluid oblique field, MHD simulation with approximately the same upstream conditions. Both models have $M_{f s}=6.1, \theta_{0}=60^{\circ}, \gamma_{c}=4 / 3$, and $\beta=1.0$. Gas and CR pressures are equal in the inflowing plasma. The time-dependent simulation used constant $\langle\kappa\rangle=0.2$. Shown are the density, gas pressure, magnetic pressure, normal velocity, tangential velocity, and CR pressure. The simulation is shown at $t / t_{d}=200,500$, and 900 , where the diffusion time, $t_{d}$, is defined in the text.

with the full momentum-dependent diffusion-advection equation. We will present elsewhere a full discussion of the strengths and weaknesses of the two-fluid model in oblique MHD shocks (Jones, Frank \& Ryu 1995) and in parallel shocks (Kang \& Jones 1995). But it is helpful at this point to offer a preliminary comparison of properties so that readers can judge the conclusions presented here.

To do this we have carried out two sets of paired two-fluid/ diffusion-advection simulations that are variations on the simulation shown in Figure 1. The MHD conditions are identical to those for Figure 1. Also, as for all the simulations presented here, $N=\frac{1}{2}$. In each of the diffusion-advection simulations the inflowing $C R$ distribution had a power-law form $f(p) \propto p^{-4.2}$, between $4.5 \times 10^{-5} \leq p \leq 2.2 \times 10^{4}$, corresponding to $\gamma_{c} \approx 1.4$. As discussed in Kang \& Jones (1991), there were 128 logarithmically spaced momentum zones in that interval. Each of the tests was carried out using a spatial resolution such that $n_{r} \approx 20$. The resolutions are generally adequate to assure that numerical convergence is good (Kang \& Jones 1991; Frank et al. 1994; Jones et al. 1995).

Although it is possible to incorporate time and spacedependent models for the closure parameters (e.g., Jones \& Kang 1992; Duffy et al. 1993) to match closely their expected evolution, we prefer to keep these tests as simple as possible in order to provide a more general characterization of the relative behaviors of the diffusion-advection and two-fluid methods. Thus our two-fluid models are based on constant values for $\gamma_{c}$ and $\langle\kappa\rangle$. For our first test $\kappa(p)=\langle\kappa\rangle=0.2$. The value of $\gamma_{c}$ near the shock in the diffusion-advection simulation did not change significantly from the inflowing value. Therefore, in the paired two-fluid calculation we used a constant $\gamma_{c}=1.4$. The structures of the flows for this pair of simulations were virtually the same with the final values of postshock $P_{c}$ agreeing to within $3 \%$, and qualitatively very similar to the shock shown in Figure 1 , where $\gamma_{c}=4 / 3$. The only significant difference with the $\gamma_{c}=4 / 3$ example shown was that the final, postshock $P_{c}$ is about $8 \%$ greater in the $\gamma_{c}=1.4$ case. In terms of the total momentum flux that difference is only roughly $2 \%$, however. For the second test we used a momentum dependent $\kappa(p)=0.2 \times p^{1 / 2}$ in the diffusion-advection simulation. This provides a diffusion-advection example in which the effective interaction lengths of the CR vary with momentum. The evolution of that shock is shown in Figure 2. The diffusion time, $t_{d}$, is not a constant in the case using the definition (2.12), but for our purposes here it is convenient and adequate to replace $\langle\kappa\rangle$ by $\kappa(p=1)=0.2$. In those terms the solution is shown at $t / t_{d}=$ 

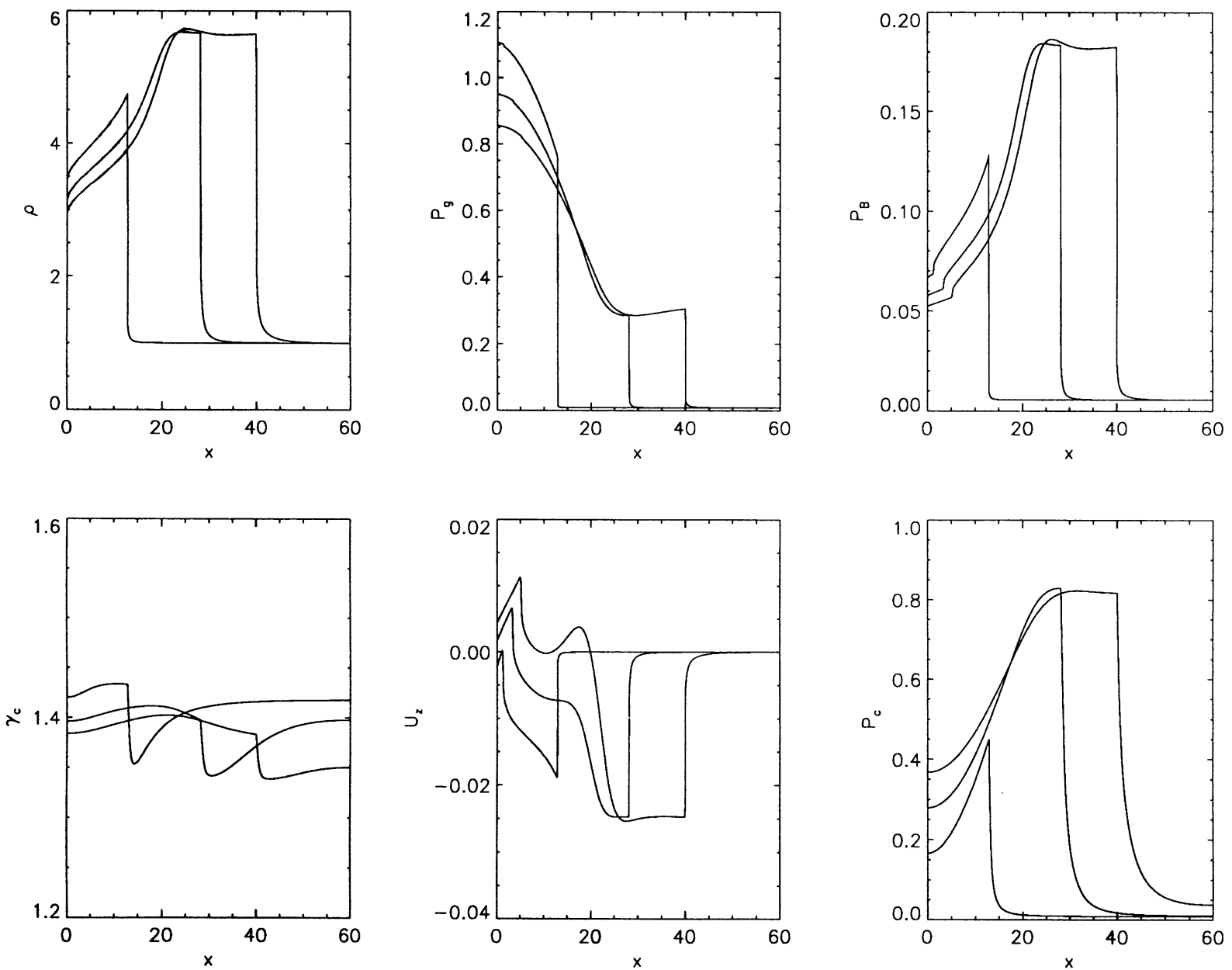

Fig. 2.-Evolution of an oblique CR-MHD shock similar to that in Fig. 1, but computed using the momentum-dependent diffusion-advection equation rather than the two-fluid technique. For this test $\kappa(p)=0.2 \times p^{1 / 2}$, while the inflowing cosmic-rays have a power-law form $f(p) \propto p^{-4 / 2}$.

$200,500,750$. To illustrate the different expense involved in this technique we mention that the simulation shown in Figure 2 required a little over 3 CPU hours on a Cray C90, compared to about 20 minutes for that shown in Figure 1. The value of $\gamma_{c}$ computed from $f(p)$ in the vicinity of the shock quickly approached $\gamma_{c} \approx 4 / 3$, as shown in Figure 2. Thus it is reasonable to directly pair this computation with the two-fluid shock already shown in Figure 1. The final properties of the two-fluid model shocks are again quite similar. $P_{c}$ in the diffusionadvection calculation is about $6 \%$ less than that found in the two-fluid computation. Again, in terms of the available momentum flux, $\bar{P}$, the difference is less than $2 \%$. The postshock flows are also reasonably similar, although we would not expect an exact match, because the evolution of the mean diffusion coefficient, $\langle\kappa\rangle$, changes the length and time scales for the flows. That should not influence equilibrium dynamical properties, but it will alter nonequilibrium ones. We conclude from these tests that two-fluid models are adequate for the purposes of this study.

\section{RESULTS}

\subsection{Steady CR-MHD Shocks: CR Acceleration Efficiency Maps}

One of our primary aims in this work is to reexamine the influence of tangential magnetic fields on the transfer of momentum to $\mathrm{CR}$ in oblique MHD shocks. All previous related discussions have pointed out that strong, oblique fields tend to inhibit acceleration efficiency, at least in shocks of modest strength. However, the physical reasons for this have not been well determined. Based on their study of perpendicular, magnetosonic shocks Jun et al. (1994) made the reasonable conjecture that efficiency was reduced because a tangential field inhibits compression by stiffening the equation of state for the fluid. But the physics of oblique CR-MHD shocks is complex and nonlinear, so this question needs further examination to be understood. For this discussion we will define the CR acceleration efficiency to be the ratio of downstream $C R$ pressure to upstream total momentum flux.

$$
\epsilon_{c}=\frac{P_{c 2}}{\bar{P}}
$$

In their study, WDV used analytical solutions to the steady CR-MHD shock equations for $M_{f}=5$ and 1 and $\theta_{0}=0^{\circ}, 30^{\circ}$, and $60^{\circ}$ to conclude that the obliquity of the field was the important acceleration inhibiting factor. To extend that effort we have completed a large set of simulations designed to map out time asymptotic values of $\epsilon_{c}$ as a function of the upstream parameters. A compact, visual way to examine how $\epsilon_{c}$ depends on the magnetic field properties in the flow is to construct maps of $\epsilon_{c}$ on a plane defined by the inflowing normal and 
tangential Alfvénic Mach numbers,

$$
\begin{gathered}
M_{x}=\frac{u_{x}}{b_{x}}=\frac{u_{x}}{b \cos \theta_{0}}=\frac{M_{g} \beta^{1 / 2}}{\cos \theta_{0}} \propto \frac{1}{B_{x}}, \\
M_{z}=\frac{u_{x}}{b_{z}}=\frac{u_{x}}{\mathrm{~b} \sin \theta_{0}}=\frac{M_{g} \beta^{1 / 2}}{\sin \theta_{0}} \propto \frac{1}{B_{z}},
\end{gathered}
$$

where $M_{g}$ is the sonic (gas) Mach number. For each map $\beta$ is held constant. Shocks with a range of shock fast-mode Mach numbers, $M_{f s}$, are obtained by varying $M_{x}$ and $M_{z}$. For this purpose it is convenient to write $M_{f}$ in terms of $\beta$ and the total Alfvénic Mach number, $M_{b}$,

$$
M_{f}^{2}=\frac{2 M_{b}^{2}}{1+\beta+\left[(1+\beta)^{2}-4 \beta \cos ^{2} \theta_{0}\right]^{1 / 2}},
$$

where

$$
\frac{1}{M_{b}^{2}}=\frac{1}{M_{x}^{2}}+\frac{1}{M_{z}^{2}}=\frac{b^{2}}{u_{x}^{2}}=\frac{1}{\beta M_{g}^{2}} .
$$

It is also useful to note that

$$
\begin{gathered}
M_{b}=M \sin 2 \theta_{0}, \\
\theta_{0}=\tan ^{-1}\left(M_{x} / M_{z}\right),
\end{gathered}
$$

and

$$
M_{z}^{2}=\frac{\rho u_{x}^{2}}{P_{B}} \underset{N=1 / 2}{\longrightarrow} \frac{\bar{P}}{P_{B}}-1-2 \beta,
$$

where

$$
M=\left(M_{x}^{2}+M_{z}^{2}\right)^{1 / 2} .
$$

Figure 3 presents such time-asymptotic efficiency maps for $\beta=20,3,0.8$, and 0.2. Each map was made from 25 simulations which uniformly covered the $\left(M_{x}, M_{z}\right)$-plane to include $2 \leq M_{f s} \leq 16$. All of the simulations have $\gamma_{c}=1.41$ and $n_{r}>$ 20 . The contours of CR acceleration efficiency, $\epsilon_{c}$, are shown as solid lines and are labeled. The contours of fast-mode shock Mach number, $M_{f s}$, are shown in dotted lines. They begin with the lowest contour set at $M_{f s}=2$ in the lower left corner, increasing in steps of 2 . Although the shocks in $\beta<1, M_{f s} \sim 2$ flows would likely be influenced by finite "Alfvén transport" effects (.e.g., Jones 1993), their inclusion here is useful in establishing trends within the CR-MHD solutions.

Some preliminary explanation should make the physics illustrated in Figure 3 easier to grasp. Note first that moving along an arc of constant radius $(M)$ from the vertical axis corresponds to sweeping through $\theta_{0}$ from $0^{\circ}$ to $90^{\circ}$. The total Alfvénic Mach number, $M_{b}$, and the fast-mode Mach number, $M_{f}$, are maximum on this arc at $\theta_{0}=45^{\circ}$. We note, as well, that for large $M_{z}$ contours of $M_{f}$ asymptote to lines $M_{x}=M_{f}$, while for large $M_{x}$ these contours asymptote to lines $M_{z}=$ $(1+\beta)^{1 / 2} M_{f}$. Thus parallel and perpendicular shocks lie only at infinity in this plane.

It is easiest to begin by examining the $\beta=20 \mathrm{map}$, since MHD effects are minimal. There one can see that both the $\epsilon_{c}$ and $M_{f s}$ contours are almost symmetric about $\theta_{0}=45^{\circ}$ and that they are almost "parallel." There is a weak tendency at large obliquity for $\epsilon_{c}$ to decrease along a Mach number contour as $\theta_{0}$ increases. This is consistent with the conclusion reached by WDV. The effect is seen to be much stronger as one looks at successively smaller $\beta$; i.e., as the field becomes dynamically more important. Consider, for example, the obliquity at which $\epsilon_{c}$ drops below 0.6 on the $M_{f s}=8$ contour. As $\beta$ decreases from 20 to 0.2 the value of the upstream obliquity at which these contours cross drops from $\theta_{0} \approx 63^{\circ}$ to $\theta_{0} \approx 47^{\circ}$. Thus, as the magnetic field becomes more dynamically significant, shocks of constant $M_{f s}$ and constant $\theta_{0}$ become less efficient CR accelerators, as found by others. Relation (4.4c) provides a clue to what is happening. Lines of constant $M_{z}$ correspond to a constant ratio of inflowing momentum flux to tangential magnetic pressure. When $\beta$ is small $P_{B} / \bar{P} \propto M_{z}^{-2}$. For $\beta<1, \epsilon_{c}$ contours in Figure 3 for high obliquity (quasi-perpendicular) shocks are closely spaced and roughly parallel to the $M_{x}$ axis, and so parallel to constant $P_{B} / \bar{P}$. Thus, it is the dynamical capacity of magnetic pressure that seems to be most significant. This is reasonable and consistent with previous conclusions, of course. The effect is important only for small to moderate $M_{f s}$, since as $M_{f s}$ becomes large $M_{z}$ is restricted to values $>(1+\beta)^{1 / 2} M_{f} \gg 1$. Then from equation (4.4c) $P_{B} / \bar{P}$ must be small for any $\beta$. This conclusion still does not discriminate among at least two possible physical reasons why the magnetic pressure plays this role. It could be, as suggested by Jun et al. (1994), just that the magnetic field reduces compression through the shock. Alternatively, it could reflect the need for the shock to do work on the field in compressing it, thus reducing the energy available to $\mathrm{CR}$.

We will describe an experiment in the following subsection that may help to resolve that issue. But, first we should comment on another aspect of the efficiency contours in Figure 3 . In this case we focus on quasi-parallel shocks, so that $M_{x}$ is relatively small. The efficiency of these shocks in the strong field $(\beta<1)$ regime seems to be higher at a given fast mode Mach number than when the field is weak. For instance, when $\theta_{0}=20^{\circ}$ and $\beta=0.2$, a $M_{f s}=4$ shock leads to $\epsilon_{c} \approx 0.55$. At the same angle this efficiency requires $M_{f s}>6$ when $\beta=20$. On the face of it, this seems to imply that the strong field has actually enhanced $\epsilon_{c}$. But that would be a misinterpretation of the results. When $\beta \ll 1 M_{f} \approx M_{b}$; i.e., the fast mode wave is much like an Alfvén wave for quasi-parallel propagation and not very compressive. The large value of $B$ that makes $c_{f} \approx b$ so large is mostly in the normal component. Using $M_{z}$ as an indicator again, we can see that the dynamical impact of the tangential field cannot be very large in this regime. Thus, the fast mode Mach number underestimates the strength of the shock as it relates to diffusive acceleration. Indeed, examination of the structures of the CR-MHD shocks here reveals that the time asymptotic compression is large $(r>4)$ and comparable to that found in large $\beta$ shocks having similar acoustic Mach number $M_{g}=M_{b} / \beta^{1 / 2}$.

This last feature should emphasize that it would be wrong to expect that the existence of strong fields necessarily leads to a reduction in the efficiency of CR acceleration in astrophysical environments. That statement seems to apply only when the tangential magnetic pressure is strong. Thus the orientation of the field is particularly important as $\beta<1$.

\subsection{Steady Solutions: The Role of Compression and Magnetic Pressure}

Diffusive shock acceleration results from simultaneous compression and diffusion of CRs. Thus the compression through the shock is itself obviously important to the efficiency of the process. But nonlinear effects complicate this simple statement. Even in CR gasdynamic shocks the acceleration efficiency rep- 

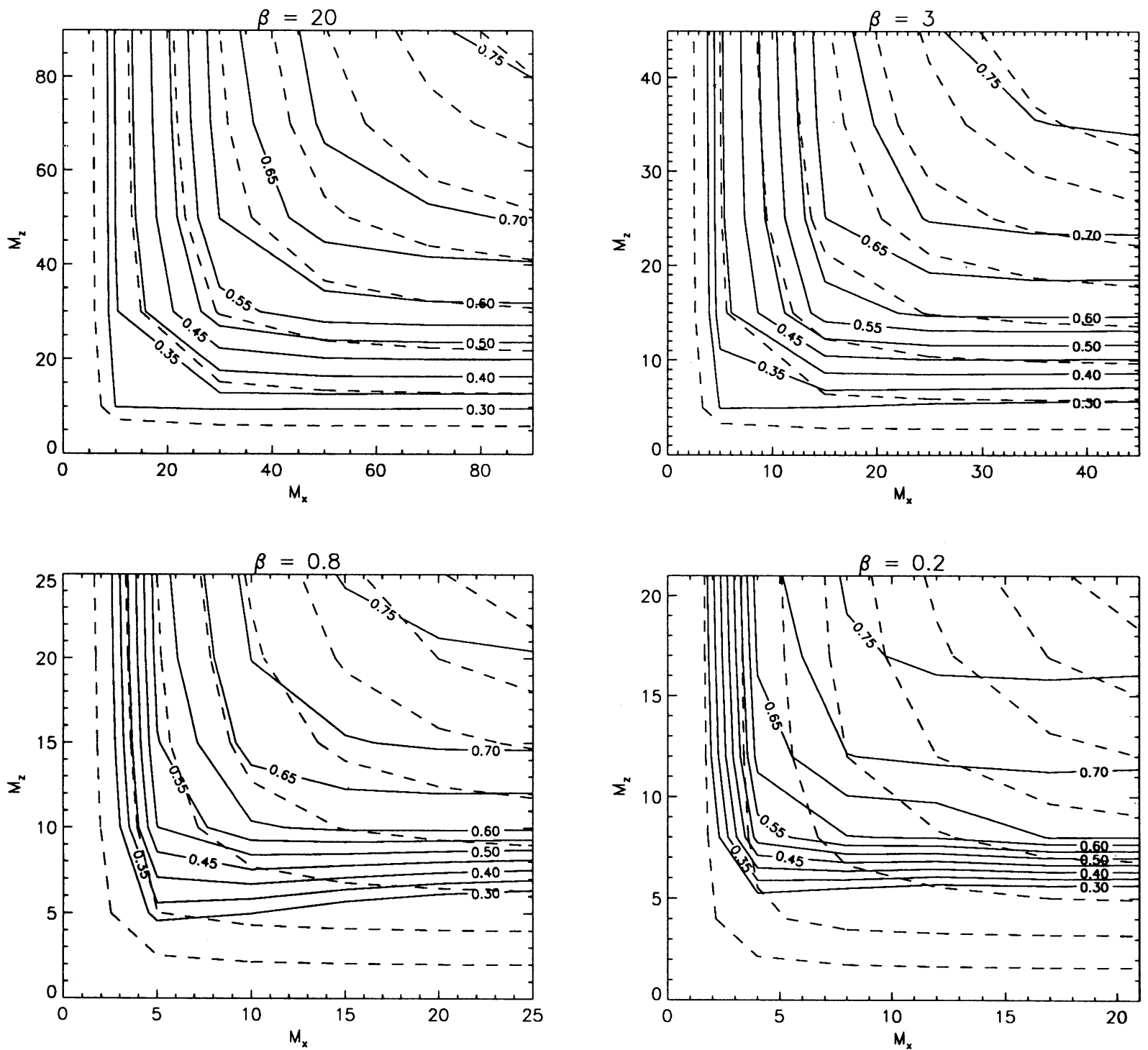

FIG. 3.-Maps of CR acceleration efficiency, $\epsilon_{c}$, as a function of normal and tangential Alfvénic piston Mach number $\left(M_{x}, M_{z}\right)$ for four different values of $\beta$. Solid lines are contours of $\epsilon_{c}$, dashed lines are contours of fast-mode shock Mach number, $M_{f s} . \epsilon_{c}$ contour levels are marked. In all the maps contours of $M_{f s}$ are separated by steps of 2, with $M_{f s}=2$ always the first contour seen in the lower left corner of the map, and $M_{f s}=16$ in the upper right. $\theta_{0}$ increase toward the $x$-axis.

resents a balance in the competition between energy input into the $\mathrm{CR}$ and the gas. The incoming momentum flux does work on both the gas and the CRs, with the partitioning dependent on the required entropy production through the structure. A general, simple relationship between total compression and efficiency does not exist. When magnetic pressure is included the situation is even more complex, since compression of the magnetic field also requires work.

To explore the relative roles of the compression itself and the work done on the magnetic field in CR-MHD shocks, we have performed a simple set of numerical experiments. We carried simulations out to dynamical steady state postshock conditions for two values of the upstream obliquity, $\theta_{0}=30^{\circ}$ and $60^{\circ}$ and two values of $\beta=5,1$. For each of these combinations we computed solutions for a range of CR adiabatic index, $4 / 3 \leq \gamma_{c} \leq 5 / 3$. By varying $\gamma_{c}$ we change the compression through the shock, since $\gamma_{c}<5 / 3$ softens the equation of state in a manner analogous to the way that the magnetic pressure stiffens it compared to the gas alone. All of the models used $M_{f}=6$.

The results are illustrated in Figure 4. Figure $4 a$ (upper left) shows the compression ratio, $r$, for the $\theta_{0}=60^{\circ}$ models as a function of $\gamma_{c}$. As we expect from previous studies (e.g., Drury \& Völk 1981; Achterberg et al. 1984; Kang \& Jones 1990) smaller $\gamma_{c}$ leads to substantially greater compression through the shock. Note, however, that the compression is independent of $\beta$ within our levels of uncertainty. As seen in Figure $4 b$ (upper right) the efficiency, $\epsilon_{c}$, does depend on $\gamma_{c}$, and thus on the compression. Note, however, that $\epsilon_{\mathfrak{c}}$ actually decreases with increasing compression; i.e., as the mixed fluid develops a softer equation of state. That trend runs counter to the compression argument given by Jun et al. (1994) regarding the role of the magnetic field. But, $\epsilon_{c}$ also depends on $\beta$. More to the point, for a fixed compression $\left(\gamma_{c}\right), \epsilon_{c}$ is reduced as the tangential magnetic field becomes stronger. The change in $\epsilon_{c}$ can be accounted for roughly in the work done on the field itself. That 

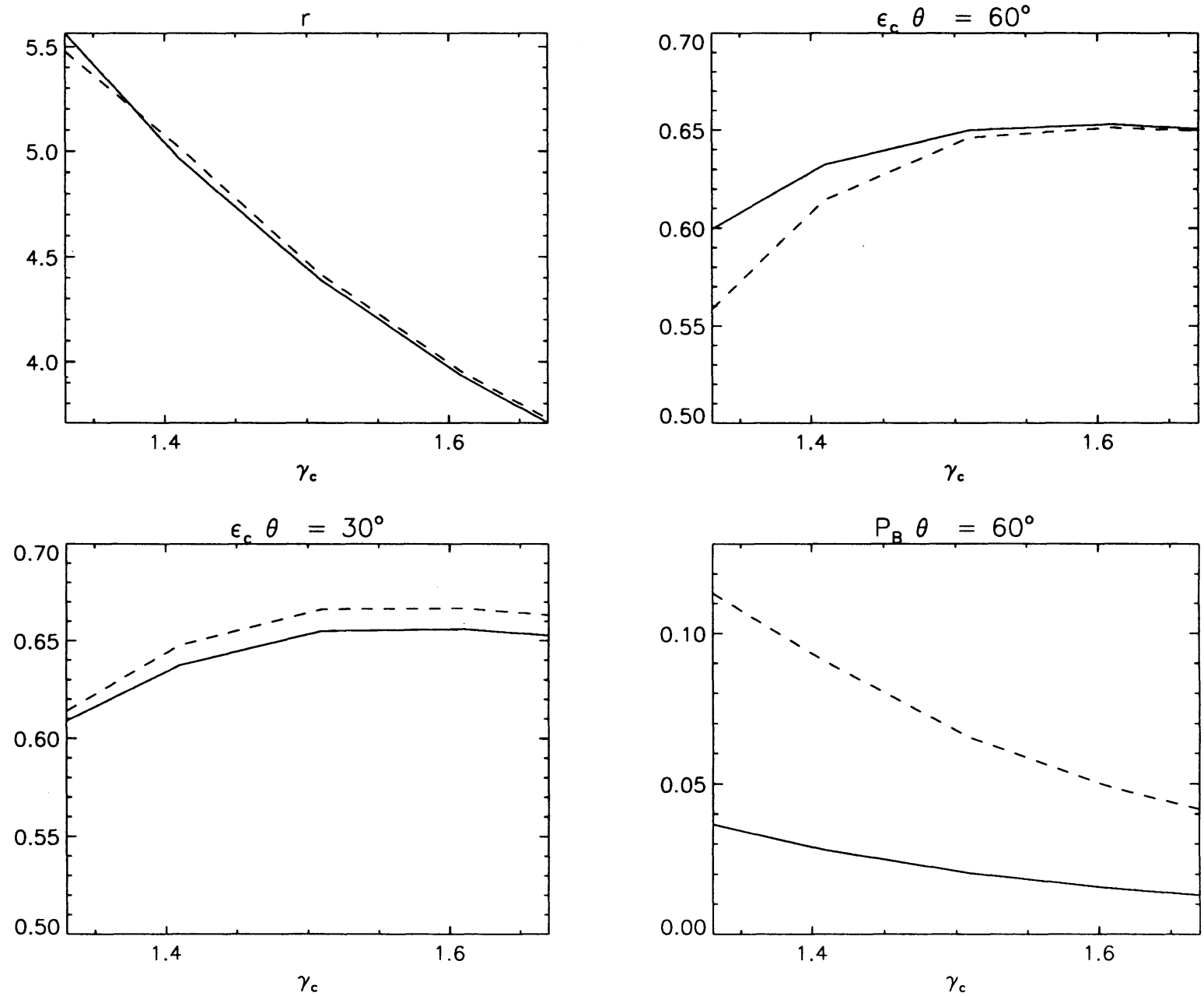

Fig. 4.-Results from a series of $M_{f s}=6$ two-fluid runs with $\beta=5$ (solid line) and $\beta=1$ (dashed line) for a range in the CR adiabatic index, $\gamma_{c}$. Upper left: compression ratio, $r=\rho_{2} / \rho_{1}$, as function of $\gamma_{c}$ for upstream $\theta_{0}=60^{\circ}$. $\beta=5$ (solid) and $\beta=1$ (dashed). Upper right: CR acceleration efficiency $\epsilon_{c}$ as function of $\gamma_{c}$ for upstream $\theta_{0}=60^{\circ}$. Lower left: CR acceleration efficiency $\epsilon_{c}$ as function of $\gamma_{c}$ for upstream $\theta_{0}=30^{\circ}$. Lower right: Normalized downstream magnetic pressure, $P_{B 2} / P$ as function of $\gamma_{c}$ for upstream $\theta_{0}=60^{\circ}$. Note that the $\beta=1$ case is always more sensitive to $\gamma_{c}$ and, therefore, to compression.

can be seen in Figure $4 d$, which shows the normalized downstream magnetic pressure, $P_{B 2} / \bar{P}$, for the same study. Two points are relevant. First, $P_{B 2} / \bar{P}$ varies in a sense opposite to $\epsilon_{c}$ as $\gamma_{c}$ varies. Second, the difference in $P_{B 2} / \bar{P}$ between the weak and strong field cases is comparable to that in $\epsilon_{c}$ for the same two cases. Thus, crudely, at least, there has been an exchange between these two quantities. But the nonlinear nature of these processes would lead us to expect a more complex situation quantitatively. Indeed, for the smaller obliquity case, $\theta_{0}=30^{\circ}$, seen in Figure $4 c$, the efficiencies for different $\beta$ are the same to within our $\sim 1 \%$ uncertainties. The magnetic pressure, $P_{B 2} / \bar{P}$, is smaller and has the same trend as in the other example. But, the low and high $\beta$ values of $P_{B 2} / \bar{P}$ differ by as much as $3 \%$. Clearly, the other energy channels (gas pressure and kinetic energy) are also involved.

\subsection{Time-dependent Effects: Nonisotropic Diffusion}

From this point we will concentrate on evolutionary aspects of CR-MHD shocks as we can understand them through twofluid models. For a variety of reasons it is likely that most astrophysically important CR shocks will not be of a steady character (see, e.g., Jones 1992).
It is well known that the time asymptotic efficiency of twofluid CR-gasdynamical shocks should be insensitive to the value or form of $\langle\kappa\rangle$ (Drury \& Völk 1981; Jones 1994). On the other hand, the detailed properties of $\langle\kappa\rangle$ do influence the rate of shock evolution and the structure of the shock. We now briefly examine those issues for oblique CR-MHD shocks. Figure 5 presents properties of two simulations differing only in the properties of $\langle\kappa\rangle$. The other properties of the flows were: $\beta=1, M_{f}=6, \gamma_{c}=1.67, \theta_{0}=60^{\circ}$. Both shocks are shown at the same dynamical time, $t$, after the shock itself has reached an equilibrium structure. The dotted line represents a shock computed with constant $\langle\kappa\rangle=0.325$, while the solid line presents a flow with an anisotropic (obliquity dependent) $\langle\kappa\rangle$ with $\left\langle\kappa_{\|}\right\rangle=1.0=10 \times\left\langle\kappa_{\perp}\right\rangle$, as defined in equation (2.11). Upstream of the shock $\langle\kappa\rangle=0.325$ in this model also. Two points are obvious from the figure. First, the final postshock states, as represented by $\rho$ and $P_{c}$, are the same in both simulations. This holds for the other state variables, as well. Second, the histories of the two shocks were different. One can trace the history by looking from left to right, since the shock moves away from the piston at the left boundary, and to the first approximation the post shock fluid is at rest and not changing 

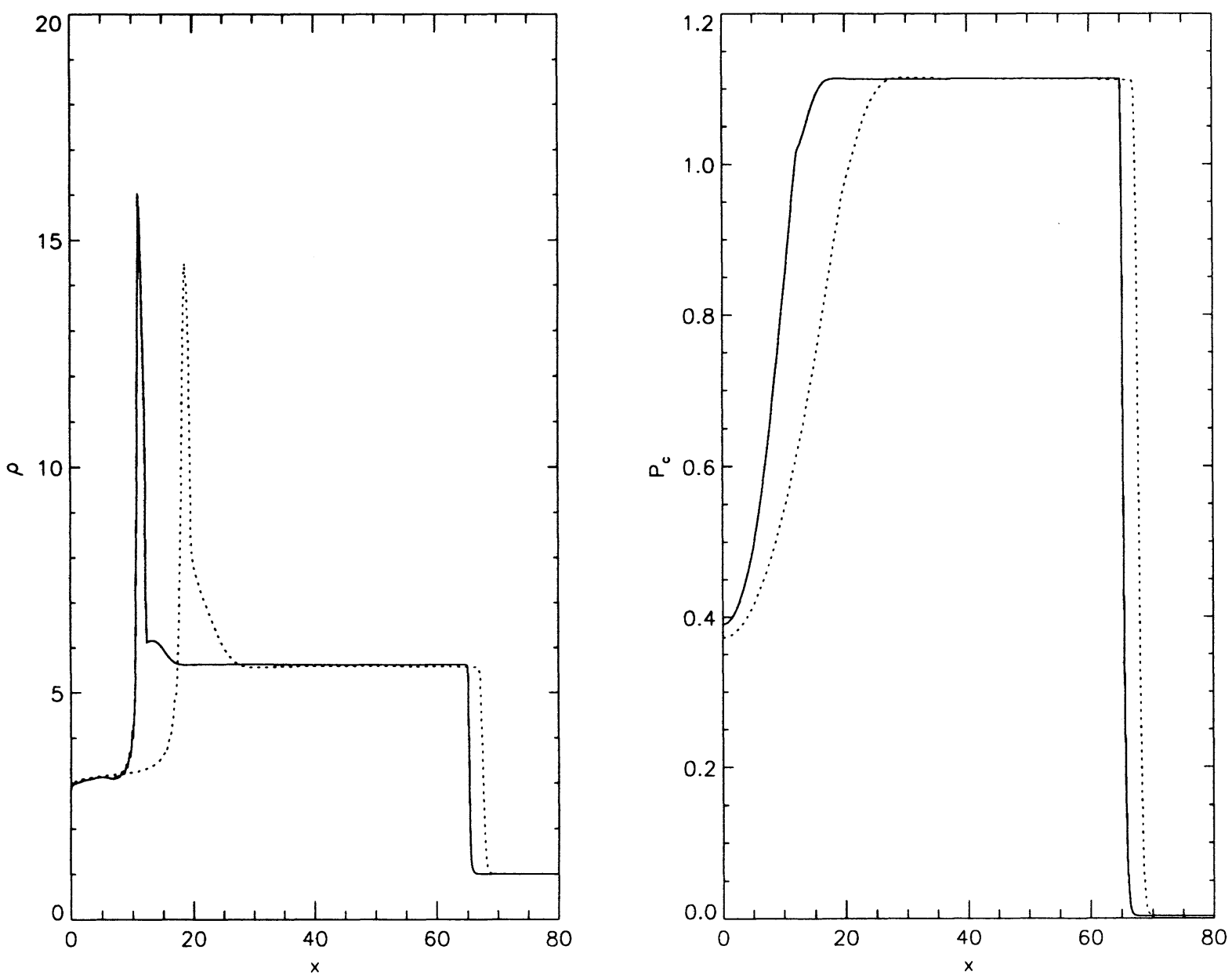

Fig. 5.-Comparison of two $\beta=1, M_{f}=6, \gamma_{c}=1.67$ two-fluid runs with different models of the CR diffusion coefficient. Dashed line shows a constant $\langle\kappa\rangle=0.325$ model. The solid line shows an obliquity dependent model (see text). Both represent the shock structures at $t / t_{d}=431$.

very fast (the next section discusses ways in which this is not quite true). The density spike identifies the approximate location of the shock when it reached dynamic equilibrium (see Fig. 1). Thus it is clear that this condition was achieved at an earlier time for the obliquity-dependent diffusion model. That results from effective differences in diffusion times for the two models. For the constant diffusion model, $t_{d}=0.325$, which matches the nominal (upstream) value for the obliquity-dependent diffusion model, using the convention established in $\S 2$. But, through the precursor of that shock $\langle\kappa\rangle$ decreases from $\langle\kappa\rangle=0.325$ to $\langle\kappa\rangle=0.1$, so that effective values of $t_{d}$ and $x_{d}$ within the precursor and postshock flows are smaller as one proceeds downstream. That accounts in part for the sharper and higher postshock density spike seen in the variable diffusion model and the earlier achievement of a steady postshock state. Furthermore, the quicker evolution to a CR-dominated postshock state with higher compression (due to the softer equation of state of the CR) explains why the position of the shock is not as far advanced for the obliquity-dependent diffusion model. The shock speed decreases as the compression increases.

The more rapid shock evolution in the obliquity-dependent diffusion model is related to the point emphasized by Jokipii (1987). He argues from test particle theory that with such diffusion models acceleration of individual particles will be quicker in highly oblique shocks, raising the possibility that particles of higher energy might be achieved in supernova remnant shocks than expected with isotropic, Bohm diffusion. Since we have not solved the diffusion-advection equation here we cannot comment directly on that issue except to make one point. More rapid acceleration also leads more quickly to the development of nonlinear feedback from the CR to the fluid. In highly oblique shocks in low $\beta$ plasmas, that would mean a prompter onset of work done by CRs on the tangential magnetic field. The net result of that, of course, is a reduction in the total work done by the flow on the CR population. Since for $\gamma_{c}>4 / 3, P_{c}$ is dominated by low-energy particles, these two behaviors are not necessarily contradictory. Clearly, a close look at this issue is warranted, however.

\subsection{Time-dependent Effects: MHD Evolution}

In Figure 6 we present the time development of a $M_{f}=10$, strong field $(\beta=1)$, CR-modified oblique $\left(\theta_{0}=60^{\circ}\right)$ MHD shocked flow. The evolution of the shock was calculated with $\gamma_{c}=1.41$ and nonisotropic diffusion using $\left\langle\kappa_{\|}\right\rangle=1$ and $\left\langle\kappa_{\perp}\right\rangle=0.1$. Three time frames are shown at $t / t_{d}=62,123$, and 185 , respectively. The plots demonstrate that the coupled CR-MHD evolution of the shock and especially the postshock flow are complex. Basic qualitative evolution of the fluid and the CRs are similar to CR-gasdynamical shocks, however, and 

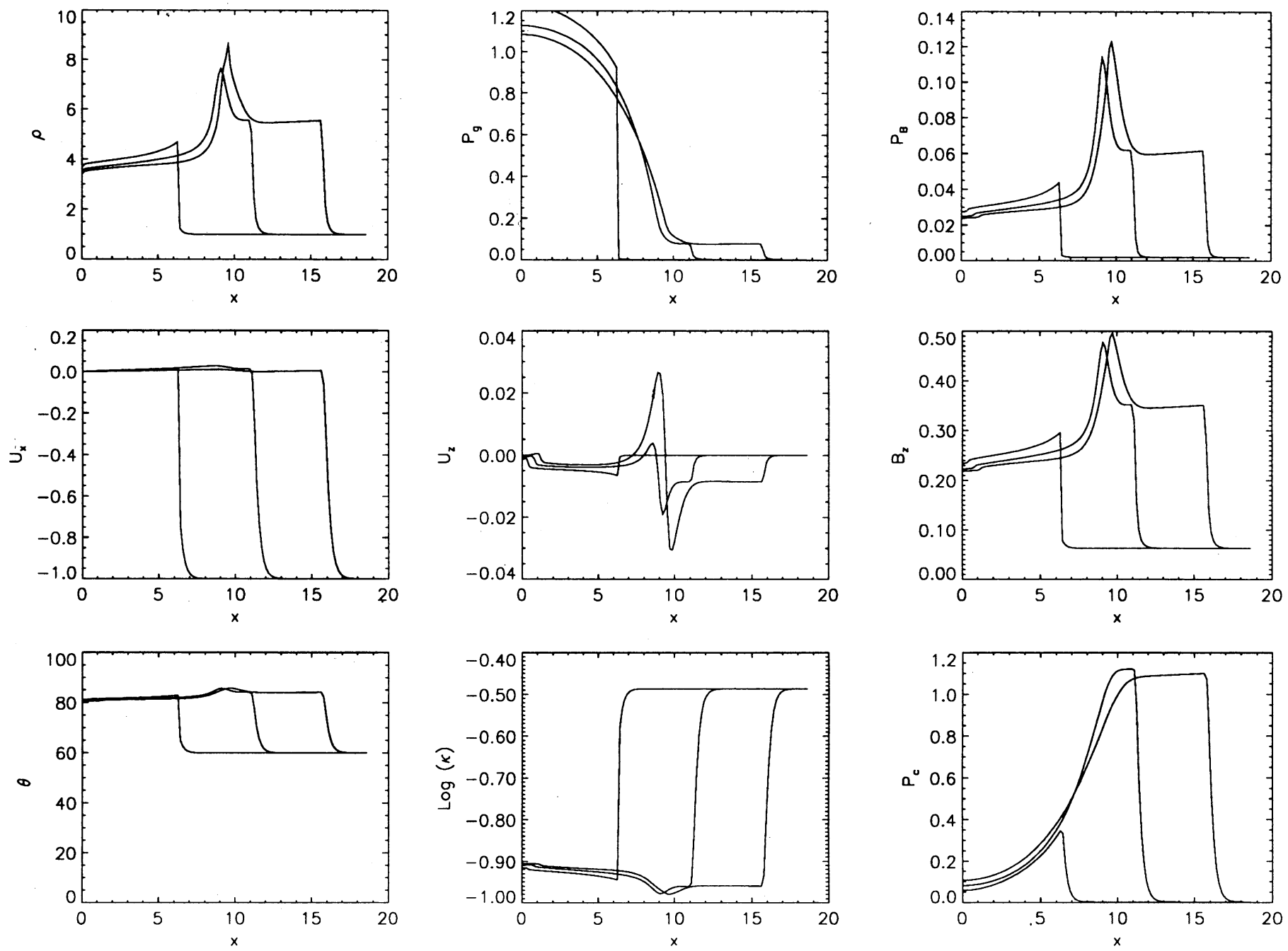

Fig. 6. - The time evolution of a model with $M_{f}=10, \theta_{0}=60^{\circ}, \gamma_{c}=1.41, P_{g 0}=0.032$, and $\beta=1.0$. $\langle\kappa\rangle$ is obliquity dependent as in Fig. 5. Structure is shown at times $t / t_{d}=62,123$, and 185 . Shown are the density, gas pressure, magnetic pressure, normal velocity, tangential velocity, tangential field, obliquity, diffusion coefficient, and CR pressure.

they have been discussed extensively in previously cited literature. We concentrate here on those features that are MHD in origin.

Through the shock the tangential magnetic field is enhanced. Mostly this is just compression, but in fast-mode shocks there is an additional, Mach number-dependent enhancement through induction generated by shear in the shock transition (e.g., Kantrowitz \& Petschek 1966). That shear is evident in the $u_{z}$ jump seen through the shock. The net tangential magnetic field change through any steady plane compression feature is given by the simple expression

$$
r_{B}=\frac{B_{z 2}}{B_{z 1}}=r\left(\frac{M_{x}^{2}-1}{M_{x}^{2}-r}\right),
$$

where $M_{x}$ refers to the condition upstream of the feature as measured in the rest frame of the feature. The factor on the right in parentheses results from shear in the transition. The shock in Figure 6 corresponds to $M_{x} \approx 27$, so $r_{B} \approx r$. But for weaker shocks, including slow shocks the shear induced term is more significant. Equation (4.5) also shows the well-known difference between fast-mode and slow-mode waves. Whereas the sign of the change in tangential field and density are the same for fast waves $\left(M_{x}^{2} \geq r>1\right)$, they are opposite for slow waves $\left(M_{x}^{2} \leq 1\right)$. This difference results from a reversal in the direction of the shear, $\left[u_{z}\right]$, relative to the normal velocity jump, $\left[u_{x}\right]$ through the feature (Kantrowitz \& Petschek 1966). Then the induction (see the magnetic terms in eq. [2.4]) also changes sign. This information helps sort out the evolution of the postshock flows seen in strong CR-MHD oblique shocks such as that shown in Figure 6. In this regard it is also useful to identify from the MHD dispersion relation that through a $\beta>1$ fast-mode compression, $\left(M_{x}\left[u_{z}\right]\right) /\left(M_{z}\left[u_{x}\right]\right)>0$. The opposite inequality applies for slow waves. For the flow described here $M_{x} / M_{z}>0$, so the normal velocity gradient and the shear velocity gradient will have opposite signs in a fast compression, but the same sign in a slow compression.

Although the shock itself in the simulations shown reaches a dynamical steady state that is not true for the postshock density (and magnetic field) spike. Such spikes result from initially strong subshocks as they adjust to new CR-dominated equilibrium conditions. At the start this feature is a fast-mode feature, since the tangential field and gas density increase together with similar form at early times. It develops, after all, from the fast-mode shock. At early times one can see that the 
shear velocity component, $u_{z}$ that leads to inductive generation of field is negative behind the (fast-mode) shock as expected from the relation just cited. But after the shock has reached an equilibrium, so that the density spike is left behind, a clear $\because$ I maximum in $u_{z}$ is present just to the left of the peak in the density spike. That signals the formation of a slow wave from the density peak. This interpretation comes from the fact that $\partial u_{x} / \partial x<0$ across almost the entire density spike, including the peak. Thus it is being slowly compressed. Hence the negative gradient in $u_{z}$ must represent a slow-mode compression. This development is even more obvious by the third and last time shown in Figure 6. By this time, in fact, the (right facing) slowmode compression has substantially reduced $B_{z}$ on its downstream side, so that the density and magnetic spikes are clearly distinct.

Subsequently, the consequences of the slow wave are striking, in fact, as seen in Figure 7. This figure shows at later times the evolution of the density spike. One sees that the magnetic spike is eventually confined to the forward (right) edge of the density spike. In fact, the forward edge of the density spike has steepened into a slow-mode shock. This is demonstrated by the sharp jump in $u_{x}$ at this point. The development of shocks in the transient density spike has not happened in any of the CR-gasdynamical shocks we have studied, so it seems to be strictly the result of the CR-MHD nature of this calculation. Although the density spike results from artificial nonequilibrium initial conditions, it may still be of much more than academic interest. Many astrophysical shocks will likely have transient properties. We have seen such density spikes in our simulations of supernova remnants (Jones \& Kang 1992) and in two-dimensional simulations of supersonic dense clumps (Jones \& Kang 1993; Jones, Kang, \& Tregillis 1994). The velocity jump at the slow-mode shock in the spike is moderately large. It actually does lead to a small amount of CR particle acceleration downstream of the main shock. That can be seen through the small "bump" in the $P_{c}$ curve at the last time shown in Figure 7. In addition, the shear and associated tangential current in the postshock flow resembles in some ways those inside the main shock. There they are accompanied by drift acceleration. For calculations in a planar geometry this does not appear, because the net energy flux vanishes (Jokipii 1982; Webb et al. 1983). However, once that symmetry is
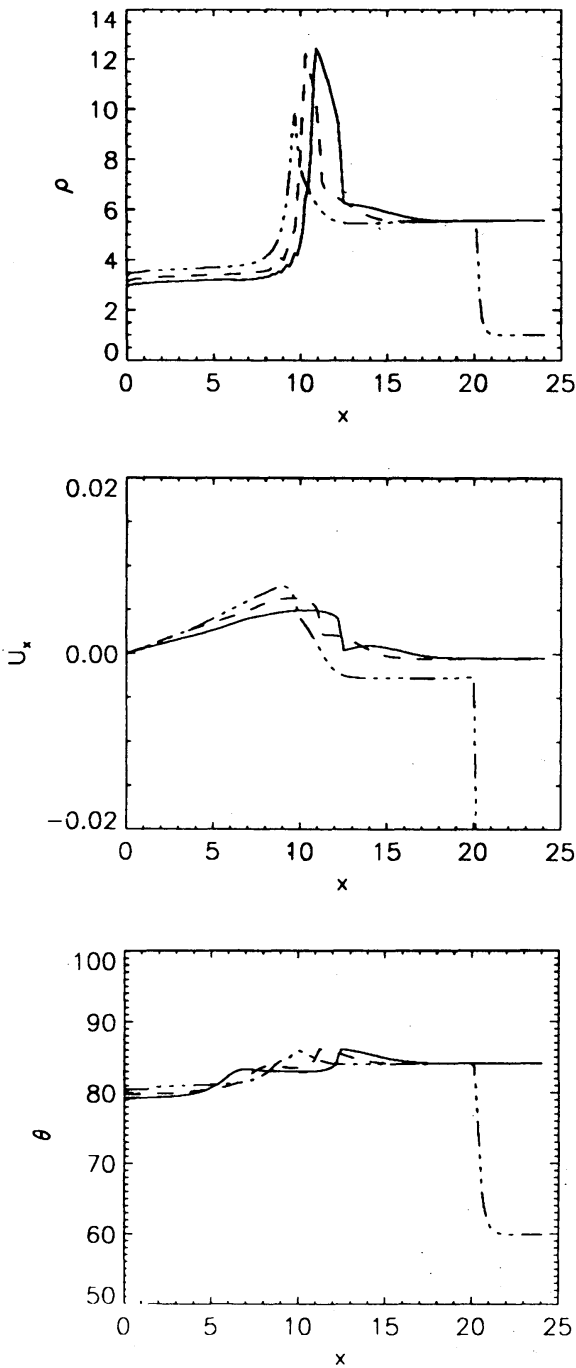
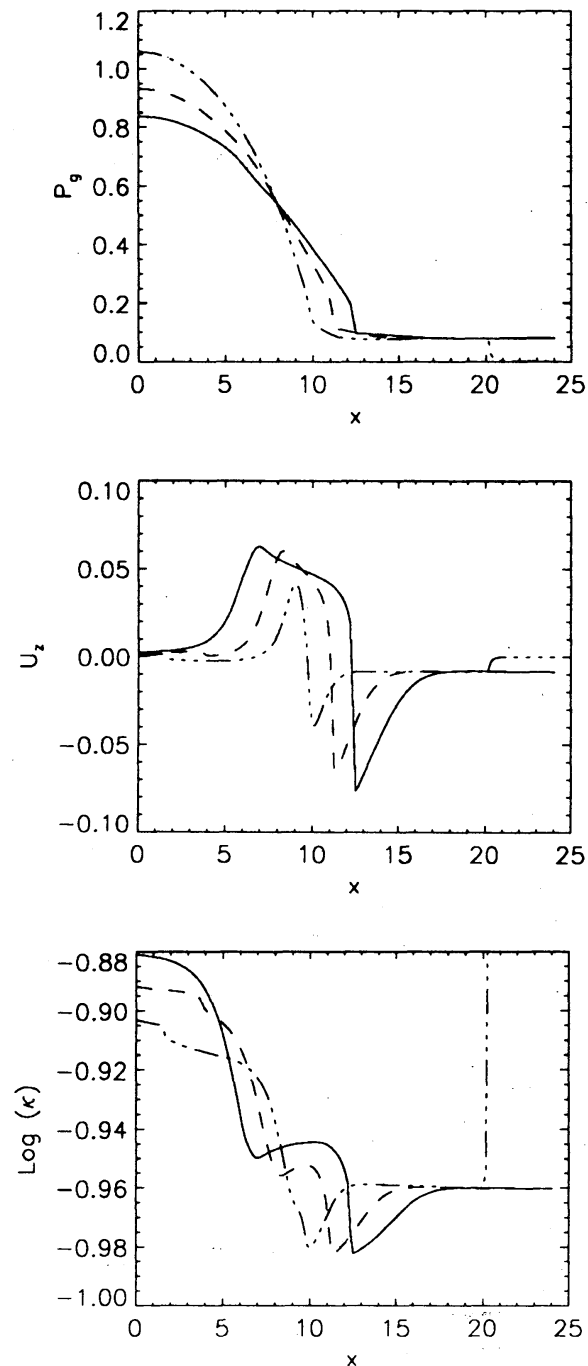
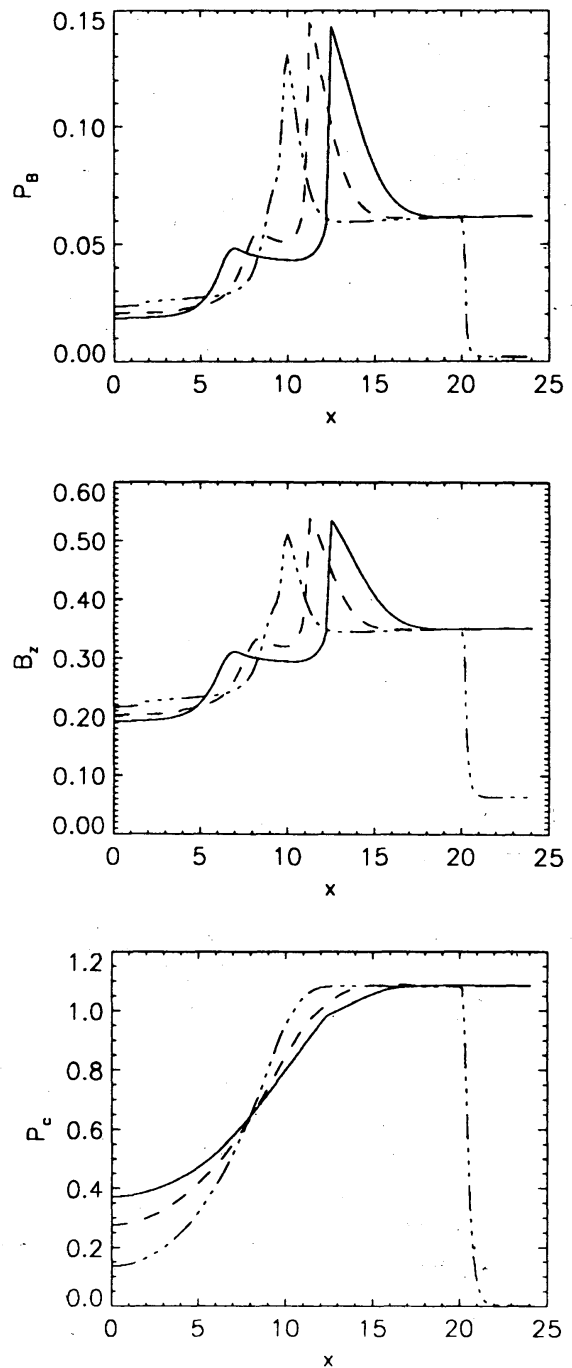

Fig. 7.-Same as Fig. 5 at times $t / t_{d}=308,615$, and 923 . Note that here we have changed scale in $x$ to focus on the evolution of the postshock density spike. 
broken, the drifts could play important roles just as they apparently due in perturbed oblique CR-MHD shocks (Decker 1988; Zank et al. 1990).

\section{SUMMARY AND CONCLUSIONS}

Utilizing a new numerical method built on the two-fluid model for diffusive shock acceleration, we have conducted a study of CR-modified oblique MHD plane shocks. We tested the code against steady state two-fluid shock solutions and against time-dependent diffusion-advection shock solutions. The results of our numerical experiments are as follows.

1. For a fixed fast-mode Mach number the particle acceleration efficiency, $\epsilon_{c}$, of time asymptotic CR-modified, quasiperpendicular MHD shocks decreases as the upstream obliquity, $\theta_{0}$, of the magnetic field increases. The dependence of $\epsilon_{c}$ on $\theta_{0}$ is greater for tangential fields whose amplification through the shock transition absorbs a significant portion of the incident momentum flux of the CR-MHD fluid. This limits the impact of the field on acceleration efficiency to moderately strong, quasi-perpendicular shocks in strong field (low $\beta$ ) plasmas. These results confirm early analytical models and recent numerical models of perpendicular, magnetosonic shocks. Our experiments, however, map the relationships between $\epsilon_{c}$ and the MHD and CR properties more fully than previous studies and demonstrate that acceleration inhibition probably results from work done on the magnetic field rather than changes in fluid compression brought about by the presence of the field, for example.

2. For quasi-parallel shocks, our results show that $\epsilon_{c}$ is higher at a given fast mode Mach number when $\beta<1$ than when $\beta>1$. While this would appear to suggest that strong fields enhance acceleration efficiency in quasi-parallel shocks, it actually results because in strong fields the quasi-parallel fast-wave speed is the Alfvén speed. The larger gas sonic Mach number is a better measure of the strength and therefore the compression of $\beta<1$ quasi-parallel shocks. Thus strong fields do not necessarily imply a reduction in $\epsilon_{c}$. It is the tangential magnetic pressure that is important.

3. The possibility of an anisotropic form for the CR diffu- sion coefficient, $\langle\kappa\rangle$, does not affect the time-asymptotic postshock state achieved by the CR-modified MHD shocks. However, the history of CR-MHD shocks can be considerably modified by different forms of $\langle\kappa\rangle$. In particular, we have examined the effect of an obliquity-dependent diffusion coefficient $\langle\kappa(\theta)\rangle$ on CR-MHD shock evolution. In these models the compression of the tangential field through the shock reduces $\langle\kappa\rangle$ is the downstream regions and reduces the time necessary to achieve a steady state. That is consistent with earlier suggestions that anisotropic diffusion in quasi-perpendicular shocks might allow individual CR particles to reach higher energies in astrophysical shocks having finite lifetimes. The fact that strong field, quasi-perpendicular shocks may at the same time become less efficient accelerators as the result of nonlinear effects needs to be studied using more complete models of diffusive acceleration. The two outcomes need not be contradictory, however, since the efficiency measures total energy exchange rather than the distribution of energies.

4. Time-dependent CR-MHD effects may become significant downstream of the shock, even once the shock itself has approached a dynamical equilibrium. In particular, if $\mathbf{C R}$ modification of the shock is sufficient to generate a postshock density spike, MHD influences in that spike can cause substantial evolution in the spike. We found that a strong postshock shear develops in response to magnetic stresses in strong, low $\beta$ oblique shocks. In more realistic flows where planar symmetry constraints are broken, this shear layer may yield additional CR acceleration through the production of drift currents. Further, a slow mode shock can form on the near edge of the postshock spike, possibly enhancing particle acceleration from the full structure.

This work by T. W. J. and A. F. was supported in part by NASA through grant NAGW-2548, the NSF through grant AST 91-00486 and AST 93-18959, and by the University of Minnesota Supercomputer Institute. This work by D. R. was supported in part by Non-Directed Research Fund, Korea Research Foundation, 1993. T. W. J. and A. F. gratefully acknowledge the hospitality of the MSI while this work was carried out.

\section{APPENDIX}

\section{FINITE DIFFERENCE ALGORITHMS}

To solve the MHD equations we have employed the total variation diminishing (TVD) method introduced by Harten (1983). The TVD method solves the vector equation (2.4) without the source vector $\boldsymbol{S}$ in conservative, finite difference form:

$$
\left(\boldsymbol{q}_{i}^{n+1}\right)^{*}=\boldsymbol{q}_{i}^{n}+\frac{\Delta t}{\Delta x}\left[\boldsymbol{f}_{i+1 / 2}^{n}(\tilde{\boldsymbol{q}})-\overline{\boldsymbol{f}}_{i-1 / 2}^{n}(\tilde{\boldsymbol{q}})\right] .
$$

As usual, $i$ is the spatial zone index; integers correspond to zone averages (centered values). The $n$ index refers to time step. The quantity

$$
\overline{\boldsymbol{f}}_{i \pm 1 / 2}^{n}(\tilde{\boldsymbol{q}})
$$

is a 4-point numerical flux function that depends on the vector $q$ and the original flux $F(q)$. The TVD prescription of the numerical flux function

$$
\overline{\boldsymbol{f}}_{i+1 / 2}^{n}(\tilde{\boldsymbol{q}})
$$

utilizes an approximate MHD Riemann solver (Roe 1981; Harten 1983; Brio \& Wu 1988; Ryu \& Jones 1995) to find estimates of the state variables at the zone boundaries. The method is an explicit, second-order, Eulerian finite difference scheme. The TVD 
scheme also applies minimal numerical viscosity to the computed solution resulting in strong shocks that are represented with only two to three zones. Contact and rotational discontinuities are generally contained within $\lesssim 10$ zones. For detailed descriptions and tests for the TVD method applied to the MHD problem see Ryu \& Jones (1995).

CR dynamical feedback on the conducting fluid is handled in a two-step process designed to preserve second-order accuracy. Gradients in $P_{c}$ accelerate and do work on the gas during a time step, $\Delta t$. To reflect that in the fluxes,

$$
\overline{\boldsymbol{f}}_{i+1 / 2}^{n}(\tilde{\boldsymbol{q}}),
$$

and thus improve the performance of the MHD Riemann solver, input Riemann solver momentum and energy densities are corrected to time-centered values by a simple estimate of the force and work produced by the gradient in $P_{c}$,

$$
\tilde{\boldsymbol{q}}=\boldsymbol{q}_{i}^{n}+\frac{\Delta t}{2} \boldsymbol{S}_{i}^{n} .
$$

Then, after the TVD step represented in equation (A1) with $\tilde{\boldsymbol{q}}$, the state vector $\boldsymbol{q}$ is updated for the effects of the source terms, $\boldsymbol{S}$; namely,

$$
\boldsymbol{q}_{i}^{n+1}=\left(\boldsymbol{q}_{i}^{n+1}\right)^{*}+\Delta t \boldsymbol{S}_{i}^{n},
$$

where $q^{*}$ represents the solution to equation (A1).

The CR pressure, $P_{c}$, is found from equation (2.1) or equation (2.2). The updating of these follow similar procedures. The CR energy equation is simpler, so we outline that. It is updated through a two-step procedure, which is also second-order accurate. In the first step we solve the Lagrangian version of equation (2.2),

$$
\frac{d E_{c}}{d t}=\frac{\partial E_{c}}{\partial t}+u \frac{\partial E_{c}}{\partial x}=\frac{\partial}{\partial x}\left(\langle\kappa\rangle \frac{\partial E_{c}}{\partial x}\right)-\gamma_{c} E_{c} \frac{\partial u}{\partial x}+S_{e},
$$

exactly as we do in the PPM two-fluid code, using a Crank-Nicholson scheme and boundary conditions explained in Jones \& Kang (1990). This is followed by a "remap" step,

$$
E_{c, i}^{n+1}=\left(E_{c, i}^{n+1}\right)^{*}-u_{i}^{n} \frac{\Delta t}{\Delta x}\left[g_{i+1 / 2}^{n}\left(E_{c}^{*}\right)-g_{i-1 / 2}^{n}\left(E_{c}^{*}\right)\right],
$$

where

$$
g_{i \pm 1 / 2}^{n}\left(E_{c}^{*}\right)=\left(E_{c, i}^{n}\right)^{*} \pm \frac{\Delta x}{2}\left(E_{c, i}^{n}\right)^{* \prime}\left(1 \pm \frac{u_{i}^{n} \Delta t}{\Delta x}\right)
$$

$E_{c}^{*}$ is the solution to equation (A5) and $\left(E_{c}\right)^{* \prime}$ is the monotone slope of $E_{c}^{*}$ (Van Leer 1974). In equations (A4)-(A6) the $x$ subscript has been dropped from $u_{x}$ to simplify notation. This operator split method of solving equation (2.2) proved to offer the best stability and accuracy among several approaches we tried in numerical experiments during code development. For the diffusion-advection scheme the Lagrangian step is discussed in Kang \& Jones (1991).

Achterberg, A. 1982, A\&A, 98, 195

Achterberg, A., Blandford, R. D., \& Periwal, V. 1984, A\&A, 98, 195

Baring, M. G., Ellison, D. C., \& Jones, F. C. 1993, ApJ, 409, 327

Berezhko, E. G., \& Krymskii, G. F. 1988, Soviet Phys.-Uspekhi, 31, 27

Bell, A. R. 1978, MNRAS, 182, 147

Blandford, R. D., \& Eichler, D. 1987, Phys. Rep., 154, 1

Brio, M., \& Wu, C. C. 1988, J. Comp. Phys., 75, 400

Chalov, S. V. 1988, Soviet Astron. Lett., 14, 114

Drury, L. O'C. 1983, Rep. Prog. Phys., 46, 973

. 1987, in Proc. 6th Internat. Solar Wind Conf., ed. U. J. Pizzo,

T. Holzer \& D. G. Sime (Boulder: NCAR/TN-360+ Proc), 521

Drury, L. O'C., \& Falle, S. A. E. G. 1986, MNRAS, 223, 353

Drury, L. O'C., \& Völk, H. J. 1981, ApJ, 248, 344

Duffy, P. 1992, A\&A, 262, 281

Duffy, P., Drury, L. O'C., \& Völk, H. J. 1993, 23rd Int. Cosmic Ray Conf.,

Calgary, 2, 259

.1994, A\&A, in press

Ellison, D. C., \& Eichler, D. 1984, ApJ, 286, 691

Falle, S. A. E. G., \& Giddings, J. R. 1987, MNRAS, 225, 399

Frank, A., Jones, T. W., \& Ryu, D. 1994, ApJS, 90, 975

Harten, A. 1983, J. Comp. Phys., 49, 357

Jeffrey, A. 1966, Magnetohydrodynamics (London: Oliver and Boyd)

Jokipii, J. R. 1982, ApJ, 255, 716

.1987, ApJ, 313,846

Jones, F. C., \& Ellison, D. C. 1991, Space Sci. Rev., 58, 259

Jones, T. W. 1992, in Particle Acceleration in Cosmic Plasmas, ed. G. P. Zank

\& T. K. Gaisser (New York : AIP), 148

. 1993, ApJ, 413, 619

1994, ApJS, 90,969

\section{REFERENCES}

Jones, T. W., Frank, A., \& Ryu, D. 1995, ApJ, in preparation

Jones, T. W., \& Kang, H. 1990, ApJ, 363, 499

1992, ApJ, 396, 575 1993, ApJ, 402, 560

Jones, T. W., Kang, H., \& Tregillis, I. L. 1994, ApJ, 432, 194

Jun, B., Clarke, D. A., \& Norman, M. L. 1994, ApJ, 429, 748

Kang, H., \& Jones, T. W. 1990, ApJ, 353, 149 1991, MNRAS, 249, 439 1995, ApJ, in press

Kang, H., Jones, T. W., \& Ryu, D. 1992, ApJ, 385, 193

Kantrowitz, A., \& Petschek, H. E. 1966, in Plasma Physics in Theory and Application, ed. W. B. Kunkel (New York: McGraw-Hill), 148

Kennel, C. F, Edmiston, J. P. \& Hada, T. 1985, in Collisionless Shocks in the Heliosphere: A Tutorial Review, ed. R. G. Stone \& B. T. Tsurutani (Washington: Amer. Geophys. Union), 1

Kirk, J. G., \& Heavens, A. F. 1989, MNRAS, 239, 995

Ostrowski, M. 1988, MNRAS, 233, 257

Parker, E. N. 1965, Planet. Sp. Sci., 13, 9

Roe, P. L. 1981, J. Comp. Phys., 43, 357

Ryu, D., \& Jones, T. W. 1995, ApJ, in press

Ryu, D., Kang, H., \& Jones, T. W. 1993, ApJ, 405, 199

Skilling, J. 1975, MNRAS, 172, 557

Slavin, J. D., \& Cox, D. P. 1992, ApJ, 392, 13

Völk, H. J., Drury, L. O'C., \& McKenzie, J. F. 1984, A\&A, 130, 19

Webb, G. M. 1983, A\&A, 127, 97

Webb, G. M., Axford, W. I., \& Terasawa, T. 1983, ApJ, 270, 537

Webb, G. M., Drury, L. O'C., \& Völk, H. J. 1986, A\&A, 160, 335(WDV)

Zank, G. P., Axford, W. I., \& McKenzie, J. F. 1990, A\&A, 233, 275 